GLOBAL DIFFERENTIAL GEOMETRIC METHODS IN ELASTICITY AND HYDRODYNAMICS

$$
\text { E.Binz }
$$

No. $115 / 90$ 


\title{
Global Differential Geometric Methods in Elasticity and Hydrodynamics
}

\author{
E. Binz \\ Dept. of Mathematics and Computer Science \\ University of Mannheim, A5 \\ D-6800 Mannheim 1, F.R.G.
}

\begin{abstract}
A formalism in the framework of global analysis and largely based on translational symmetry is used to relate in a one-to-one correspondence force densities of elasticity and constitutive laws. The customary setting of elasticity developed e.g. in [17] is included in our approach. Moreover, a structural viscosity coefficient can be naturally introduced and a dynamical setting based on d'Alembert's principle yields a Navier-Stokes type of equation.
\end{abstract}

\section{Introduction}

The purpose of this review article is to show a formalism in the framework of global analysis, which relates in a one-to-one correspondence two notions in the theory of elasticity and hydrodynamics. This correspondence is given by a Neumann problem converting force densities into constitutive maps which in turn characterize constitutive laws. This method is largely based on translational symmetry. As we will see below the customary setting of elasticity developed e.g. in [17] is included in our approach. Moreover, this formalism offers a natural way to introduce a structural viscosity coefficient.

To describe in short what we mean by a constitutive law, we begin by looking at a moving deformable bounded body in $\mathbb{R}^{n}$. The material should constitute a deformable medium. The medium forming the boundary may differ from the one forming the inside of the body. We make the geometric assumption that at any time the body is diffeomorphic to a compact, connected, oriented and smooth manifold of fixed dimension with (oriented) boundary. The boundary needs not to be connected. These assumptions allow us to think of a standard body $M$. Consequently a configuration is a smooth embedding from $M$ into $\mathbb{R}^{n}$. The configuration space is hence the collection $E\left(M, \mathbb{R}^{n}\right)$ of all smooth embeddings of $M$ into $\mathbb{R}^{n}$. This set equipped with Whitney's $C^{\infty}$-topology is a Fréchet manifold 
(cf. [8]). A smooth motion of the body in $\mathbb{R}^{n}$, therefore, is described by a smooth curve in $E\left(M, \mathbb{R}^{n}\right)$. (The calculus on Fréchet manifolds adopted in the sequel is the one presented in [8], which in our setting coincides with the one developed in [9].)

The physical qualities of the deforming medium enter certainly the work $F(J)(L)$ needed to deform (infinitesimally) the material at any configuration $J \in E\left(M, \mathbb{R}^{n}\right)$ in any direction $L$. The directions are tangent vectors to $E\left(M, \mathbb{R}^{n}\right)$ and thus are nothing else but maps in $C^{\infty}\left(M, \mathbb{R}^{n}\right)$ and vice versa.

In the following we take $F$, which is assumed to be a smooth one-form on $E\left(M, \mathbb{R}^{n}\right)$, as the basic entity of our notion of a constitutive law. In specifying the notion of a constitutive law somewhat further, we require that the constitutive properties should not be affected by the particular location of the body in $\mathbb{R}^{n}$, hence $F$ has to be invariant under the operation of the translation group $\mathbb{R}^{n}$ of the real vector space $\mathbb{R}^{n}$. In addition to this we require that $F(J)(L)=0$, for any constant map $L$, and any $J \in E\left(M, \mathbb{R}^{n}\right)$, a condition which will be interpreted a few lines below.

The forms $F$, which have these two properties, can be regarded as one-forms on $\left\{\mathrm{d} J \mid J \in E\left(M, \mathbb{R}^{n}\right)\right\}$, where $\mathrm{d} J$ is the differential of $J$. This reduced configuration space is a Fréchet manifold as well and is denoted by $E\left(M, \mathbb{R}^{n}\right) / \mathbb{R}^{n}$. It can naturally be identified via the differential operator $d$ with the space of all those configurations, which have a fixed center of mass. A smooth one-form on $E\left(M, \mathbb{R}^{n}\right) / \mathbb{R}^{n}$ will be denoted by $F_{\mathbb{R}^{n}}$. It describes the work if the center of mass is fixed. We thus deal with one-forms of the type $F=\mathrm{d}^{*} F_{\mathbb{R}^{n}}$. The space $E\left(M, \mathbb{R}^{n}\right) / \mathbb{R}^{n}$ admits a natural metric $\mathcal{G}$ of an $L_{2}$-type, which is $\mathrm{SO}(n)$-invariant and closely related to the classical Dirichlet integral.

A one-form $F$ being of this type is called a constitutive law, provided $F_{\mathbb{R}^{n}}$ admits an integral representation. It turns out that any constitutive law $F$ is determined by some smooth map $\mathcal{H} \in C^{\infty}\left(E\left(M, \mathbb{R}^{n}\right) / \mathbb{R}^{n}, C^{\infty}\left(M, \mathbb{R}^{n}\right)\right)$, called a constitutive map.

Hence in our setting we characterize the medium as far as the internal physical properties are encodable in the function $\mathcal{H}$. This constitutive function $\mathcal{H}$ determines at any $\mathrm{d} J \in E\left(M, \mathbb{R}^{n}\right) / \mathbb{R}^{n}$ two smooth force densities $\Phi(\mathrm{d} J)$ and $\varphi(\mathrm{d} J)$ linked to $\mathcal{H}(\mathrm{d} J)$ by the following system of equations:

$$
\Delta(J) \mathcal{H}(\mathrm{d} J)=\Phi(\mathrm{d} J) \text { and } \quad \mathrm{d} \mathcal{H}(\mathrm{d} J)(\mathbf{n})=\varphi(\mathrm{d} J) .
$$

Here $\Delta(J)$ is the Laplacian determined by the pull back under $J$ of a fixed scalar product on $\mathbb{R}^{n}$ and $\mathbf{n}$ is the positively oriented unit normal of $\partial M$ in $M$. The integrability condition necessary to solve this Neumann problem of which the force densities are given and the function $\mathcal{H}$ is the unknown, is equivalent with the requirement that

$$
F(J)(z)=0 \quad \forall J \in E\left(M, \mathbb{R}^{n}\right) \text { and } \forall z \in \mathbb{R}^{n} .
$$

This condition can be interpreted by saying that the resulting forces acting upon the fixed center of mass vanish. The constitutive map $\mathcal{H}$ determines a stress tensor 
$T$ given by

$$
T(J)(X, Y):=<\mathrm{d} \mathcal{H}(J) X, \mathrm{~d} J Y>\quad \forall J \in E(M, N)
$$

$X, Y$ vary among all smooth vector fields on $M$. Vice versa any stress tensor yields a constitutive map via the force densities mentioned above, if $\operatorname{dim} M=n$.

Since $F$ is also affected by the material forming the boundary, we treat in an analogous way the boundary material and exhibit in analogy to $\mathcal{H}$ a characteristic constitutive map $\eta$ (which in turn determines its own force density along $\partial M$ ) which differs from $\mathrm{d} \mathcal{H}(\mathrm{d} J)(\mathbf{n})$. This observation allows us to decode the influence of the whole body on the physical quality of the boundary material. What we have described so far is presented in the first six sections.

In Sect. 7 we show that $\mathcal{H}$ and $\eta$ are structured in the sense that in both of them the work needed to deform volume, area and shape of the body and the boundary, respectively, is naturally encoded. This observation is illustrated on two bubble models in Sect. 8. It will be apparent that if the qualities of the material depend on the shape, then the bubble (if it exists at all !) has to be an immersed torus. In Sect. 9 we touch the influence of the action of the rotation group on the configuration space.

The remaining sections deal with a dynamics for boundary-less bodies which yield a Navier-Stokes type of equations. This dynamics is based on d'Alembert's principle, where the constitutive law is given by the virtual work and thus determines the deviation from the free motions. The key to this application of the formalism developed earlier is to generalize the notion of a constitutive law in such a way that it depends not only on configurations but also on velocities.

In the first three appendices some technical tools are developed. The last appendix deals with a natural metric on the space of embeddings. This metric is based on a mass density. Its geodesics describe the collections of free motions of the pointlike material particles of the body in the ambient space.

\section{Configuration and Phase Space}

Let us think of a deformable material body moving and deforming in the Euclidean space $\mathbb{R}^{n}$. We make the geometric assumption that at any time the body maintains the shape of a $m$-dimensional, compact, connected, oriented and smooth manifold with (oriented) boundary. We assume $m \leq n$. The boundary shall not necessarily be connected. The physical qualities of the medium forming the boundary may differ from the ones forming the inside of the body.

Hence a configuration is a smooth embedding $J: M \longrightarrow \mathbb{R}^{n}$ and the space of configurations is $E\left(M, \mathbb{R}^{n}\right)$, the collection of all smooth embeddings of $M$ into $\mathbb{R}^{n}$, endowed with the $C^{\infty}$-topology. It is thus a Fréchet manifold (cf. [8] or [14]). 
Clearly each $J \in E\left(M, \mathbb{R}^{n}\right)$ induces a smooth embedding $J \mid \partial M: \partial M \longrightarrow \mathbb{R}^{n}$ of the boundary i.e. a configuration of the boundary $\partial M$ of the body. Let us denote the collection of all smooth embeddings of $\partial M$ into $\mathbb{R}^{n}$ by $E\left(\partial M, \mathbb{R}^{n}\right)$. The latter space endowed with the $C^{\infty}$-topology is a Fréchet manifold, too. In fact it is a principal bundle with the diffeomorphism group of $M$ as structure group (cf. [6]).

The phase space of the body is

$$
T E\left(M, \mathbb{R}^{n}\right)=E\left(M, \mathbb{R}^{n}\right) \times C^{\infty}\left(M, \mathbb{R}^{n}\right) .
$$

Here $C^{\infty}\left(M, \mathbb{R}^{n}\right)$ is the Fréchet space consisting of the collection of all smooth maps from $M$ to $\mathbb{R}^{n}$, endowed with the $C^{\infty}$-topology. It contains $E\left(M, \mathbb{R}^{n}\right)$ as an open subset. Proceeding for $\partial M$ as for $M$ we obtain $E\left(\partial M, \mathbb{R}^{n}\right)$ as an open subset of the Fréchet space $C^{\infty}\left(\partial M, \mathbb{R}^{n}\right)$ (cf. [14]). Its tangent bundle is obviously trivial, too, i.e. the phase space of the boundary is $E\left(\partial M, \mathbb{R}^{n}\right) \times C^{\infty}\left(\partial M, \mathbb{R}^{n}\right)$.

In the sequel of these notes we write $O_{\partial}$ instead of $\left\{J|\partial M| J \in E\left(M, \mathbb{R}^{n}\right)\right\}$. The map assigning to any $J \in E\left(M, \mathbb{R}^{n}\right)$ its restriction $J \mid \partial M$ is called $R$.

On the configuration space of the body we have two natural actions, namely

$$
t: E\left(M, \mathbb{R}^{n}\right) \times \mathbb{R}^{n} \longrightarrow E\left(M, \mathbb{R}^{n}\right)
$$

and

$$
s: \mathrm{SO}(n) \times E\left(M, \mathbb{R}^{n}\right) \longrightarrow E\left(M, \mathbb{R}^{n}\right)
$$

assigning to each $J \in E\left(M, \mathbb{R}^{n}\right)$ and each $z \in \mathbb{R}^{n}$ the embedding $J+z$ and $g \circ J$ for each $g \in \mathrm{SO}(n)$, respectively. These actions reflect the translational and the rotational symmetry on $E\left(M, \mathbb{R}^{n}\right)$ respectively. $t$ and $s$ extend obviously to $C^{\infty}\left(M, \mathbb{R}^{n}\right)$. The groups $\mathbb{R}^{n}$ and $\mathrm{SO}(n)$ both act accordingly on $E\left(\partial M, \mathbb{R}^{n}\right)$. These actions restrict to $O_{\partial}$ and obviously both also extend to $C^{\infty}\left(\partial M, \mathbb{R}^{n}\right)$.

The orbit spaces of the respective actions of the translation group $\mathbb{R}^{n}$ are denoted by $C^{\infty}\left(M, \mathbb{R}^{n}\right) / \mathbb{R}^{n}, C^{\infty}\left(\partial M, \mathbb{R}^{n}\right) / \mathbb{R}^{n}, E\left(M, \mathbb{R}^{n}\right) / \mathbb{R}^{n}, E\left(\partial M, \mathbb{R}^{n}\right) / \mathbb{R}^{n}$ and $O_{\partial} / \mathbb{R}^{n}$.

The nature of these spaces is easily understood if we introduce for any map $L \in C^{\infty}\left(M, \mathbb{R}^{n}\right)$ the differential $\mathrm{d} L$ which is locally given by the Fréchet derivative. The tangent map $T L$ of $L$ is, therefore, $(L, \mathrm{~d} L)$. The respective notion of $l \in$ $C^{\infty}\left(\partial M, \mathbb{R}^{n}\right)$ is introduced accordingly. Hence the orbit spaces mentioned above are nothing else but spaces of differentials of the elements of those spaces, on which $\mathbb{R}^{n}$ acts.

For our later investigations we observe that $M$ and $\partial M$ inherit via respective embeddings into $\mathbb{R}^{n}$ some basic geometric structures described in the first appendix. In particular each $J \in E\left(M, \mathbb{R}^{n}\right)$ and each $j \in E\left(\partial M, \mathbb{R}^{n}\right)$ yield, by pulling 
back the scalar product $<,>$ of $\mathbb{R}^{n}$ to $M$, the two Riemannian metrics $m(J)$ and $m(j)$ on $M$ and $\partial M$ respectively. In turn each $J$ and each $j$ also define in a unique way Riemannian volume elements $\mu(J)$ on $M$ and $\mu(j)$ on $\partial M$ respectively. In case $j:=J \mid \partial M$ these are related to each other by $i_{\mathbf{n}} \mu(J)=\mu(j)$ with $\mathbf{n}$ the positively oriented unit normal along $\partial M \subset M$. Clearly, this unit normal $\mathbf{n}$ depends on $J$ !

\section{The Notion of Work and Fixing the Center of Mass}

We will characterize the type of the material constituting the body $M$ in so far as it affects the work caused by an infinitesimal distortion of $M$ (cf. [13], [12],[3],[7]). This idea is formalized by giving a smooth one-form on $E\left(M, \mathbb{R}^{n}\right)$, i.e. a smooth map

$$
F: E\left(M, \mathbb{R}^{n}\right) \times C^{\infty}\left(M, \mathbb{R}^{n}\right) \longrightarrow \mathbb{R}
$$

which varies linearly in the second argument. We interpret $F(J)(L)$ as the work done if $M$ is distorted by $L \in C^{\infty}\left(M, \mathbb{R}^{n}\right)$ at the configuration $J \in E\left(M, \mathbb{R}^{n}\right)$. We call the medium described by $F$ a smoothly deformable medium.

In order to describe only internal qualities of the medium we expose $F$ to the translational symmetry and require that

$$
F(J+z)=F(J), \quad \forall J \in E\left(M, \mathbb{R}^{n}\right), \quad \forall z \in \mathbb{R}^{n} .
$$

This means that the work caused by (only internal) physical processes does not depend on the particular location of $J(M)$ within $\mathbb{R}^{n}$. Moreover, we require that a constant distortion by any $z \in \mathbb{R}^{n}$ causes no work. It is a considerably weaker condition than to assume that the internal force densities only depend on the metric relations between the particles of the body (cf. [21], where problems of isometric deformations are studied). Formally expressed we impose the further restriction:

$$
F(J)(z)=0, \quad \forall J \in E\left(M, \mathbb{R}^{n}\right), \quad \forall z \in \mathbb{R}^{n}
$$

on $F$. This restriction is very fundamental in our development. We will interpret it further below. To implement the possibility of extracting force densities from our basic notion of work, we need a little more structure associated with our forms satisfying (1) and (2). We will do this in the next section. But first we investigate the notion of work more closely:

To do so let us denote the collection of all smooth $\mathbb{R}^{q}$-valued one-forms on a manifold $Q$ (finite or infinite dimensional!) by $A^{1}\left(Q, \mathbb{R}^{q}\right)$. From Sect. 1 it is clear that any $F \in A^{1}\left(E\left(M, \mathbb{R}^{n}\right), \mathbb{R}\right)$ satisfying (1) and (2) is of the form

$$
F(J)(L)=F_{\mathbb{R}^{n}}(\mathrm{~d} J)(\mathrm{d} L), \quad \forall J \in E\left(M, \mathbb{R}^{n}\right) \quad \text { and } \quad \forall L \in C^{\infty}\left(M, \mathbb{R}^{n}\right),
$$

where $F_{\mathbb{R}^{n}} \in A^{1}\left(E\left(M, \mathbb{R}^{n}\right) / \mathbb{R}^{n}, \mathbb{R}\right)$. Instead of $(3)$ we write $F=\mathrm{d}^{\star} F_{\mathbb{R}^{n}}$.

By introducing the center of mass for any $J \in E\left(M, \mathbb{R}^{n}\right)$ we can interpret the forms in $A^{1}\left(E\left(M, \mathbb{R}^{n}\right) / \mathbb{R}^{n}, \mathbb{R}\right)$ as follows: Let us choose a map o $E$ $C^{\infty}\left(E\left(M, \mathbb{R}^{n}\right), \mathbb{R}\right)$, for which the mass $\mathbf{m}$ defined by 
6 E. Binz

$$
\mathbf{m}(J):=\int_{M} o(J) \mu(J), \quad \forall J \in E\left(M, \mathbb{R}^{n}\right)
$$

is positive. The center of mass $z_{0}(J)$ is given by the equation

$$
\mathbf{m}(J) \cdot z_{0}(J)=\int_{M} o(J) \cdot J \mu(J)
$$

or equivalently

$$
\mathbf{m}(J)<z_{0}(J), z>=\int_{M} o(J)<J, z>\mu(J),
$$

for all $J \in E\left(M, \mathbb{R}^{n}\right)$ and all $z \in \mathbb{R}^{n}$. Let $E_{0}\left(M, \mathbb{R}^{n}\right)$ be the collection of all $J \in E\left(M, \mathbb{R}^{n}\right)$, for which the center of mass $z_{0}(J)$ is fixed, e.g. $z_{0}(J)=0$. Then we realize $E\left(M, \mathbb{R}^{n}\right) / \mathbb{R}^{n}$ as a space of configurations via the map

$$
\mathrm{d}: E_{0}\left(M, \mathbb{R}^{n}\right) \longrightarrow E\left(M, \mathbb{R}^{n}\right) / \mathbb{R}^{n},
$$

sending any $J$ in the domain into $\mathrm{d} J$. It is a diffeomorphism. Moreover, we observe

$$
E_{0}\left(M, \mathbb{R}^{n}\right) \oplus \mathbb{R}^{n}=E\left(M, \mathbb{R}^{n}\right)
$$

(if $z_{0}(J)=0$, then this splitting is orthogonal with respect to (4)) and hence that

$$
T E\left(M, \mathbb{R}^{n}\right)=T E_{0}\left(M, \mathbb{R}^{n}\right) \oplus T \mathbb{R}^{n} .
$$

Having the meaning of $E_{0}\left(M, \mathbb{R}^{n}\right)$ in mind, equation (3) tells us that $F_{\mathbb{R}^{n}}$ can be interpreted as the work in case the center of mass is kept fixed.

\section{The Notion of a Constitutive Law and the Dirichlet Integral}

The purpose of this section is to define the notion of a constitutive law. However, the additional structure mentioned above relies on an integral representation of a one-form $F_{\mathbb{R}^{n}}$ on $E\left(M, \mathbb{R}^{n}\right) / \mathbb{R}^{n}$. To prepare this notion we need to represent $\mathbb{R}^{n}$-valued one-forms relative to the differential of embeddings. To do so let $\gamma \in A^{1}\left(M, \mathbb{R}^{n}\right)$ and $J \in E\left(M, \mathbb{R}^{n}\right)$ be given and let us consider the two-tensor $T(\gamma, \mathrm{d} J)$ determined by $\langle\gamma X, \mathrm{~d} J Y\rangle$ for all $X, Y \in \Gamma T M$. This-two tensor yields a unique strong bundle map $A(\gamma, \mathrm{d} J)$ of $T M$ defined by

$$
T(\gamma, \mathrm{d} J)(X, Y)=m(J)(A(\gamma, \mathrm{d} J) X, Y), \quad \forall X, Y \in \Gamma T M
$$

From this equation we read off :

$$
\gamma X=\mathrm{d} J A(\gamma, \mathrm{d} J) X+(\gamma X)^{\perp}, \quad \forall X \in \Gamma T M
$$

with $(\gamma X)^{\perp}$ being pointwise orthogonal to $\mathrm{d} J T M$ (which appears if $\operatorname{dim} M<n$ ). 
Let us rewrite $\bigcup_{p \in M} T_{J(p)} \mathbb{R}^{n}$ by $T \mathbb{R}^{n} \mid J(M)$ which is the restriction of the tangent bundle $T \mathbb{R}^{n}$ to $J(M)$. Hence we find a bundle map

$$
c(\gamma, \mathrm{d} J): T \mathbb{R}^{n}\left|J(M) \longrightarrow T \mathbb{R}^{n}\right| J(M)
$$

mapping the vector space $\mathrm{d} J T_{p} M$ into the normal space of $\mathrm{d} J T_{p} M$ and vice versa for each $p \in M$. Without loss of generality we may assume that $c(\gamma, \mathrm{d} J)(p)$ : $\mathbb{R}^{n} \longrightarrow \mathbb{R}^{n}$ is skew symmetric with respect to $<,>$ for each $p \in M$. We therefore arrive at our desired unique representation:

$$
\gamma X=c(\gamma, \mathrm{d} J) \mathrm{d} J X+\mathrm{d} J A(\gamma, \mathrm{d} J) X
$$

For any two one-forms $\gamma_{1}, \gamma_{2} \in A^{1}\left(M, \mathbb{R}^{n}\right)$ along an embedding $J \in E\left(M, \mathbb{R}^{n}\right)$ we define the dot product of $\gamma_{1}$ and $\gamma_{2}$ relative to $J$ by

$$
\gamma_{1} \cdot \gamma_{2}:=-\frac{1}{2} \operatorname{Tr} c\left(\gamma_{1}, \mathrm{~d} J\right) c\left(\gamma_{2}, \mathrm{~d} J\right)+\operatorname{Tr} A\left(\gamma_{1}, \mathrm{~d} J\right) \cdot \tilde{A}\left(\gamma_{2}, \mathrm{~d} J\right)
$$

Here $\tilde{A}\left(\gamma_{2}, \mathrm{~d} J\right)$ is the adjoint of $A\left(\gamma_{1}, \mathrm{~d} J\right)$ formed fiber-wise with respect to $m(J)$. Associated with this product is a type of a scalar product $\mathcal{G}(J)$ on $A^{1}\left(M, \mathbb{R}^{n}\right)$ defined by

$$
\mathcal{G}(J)\left(\gamma_{1}, \gamma_{2}\right):=\int_{M} \gamma_{1} \cdot \gamma_{2} \mu(J)
$$

We call the right hand side of (7) the Dirichlet-integral (cf. [5]), it is $\mathrm{SO}(n)$ invariant.

We equip $A^{1}\left(M, \mathbb{R}^{n}\right)$ with the $C^{\infty}$-topology (cf. [8]). The real number $\mathcal{G}(\mathrm{d} J)\left(\gamma_{1}, \gamma_{2}\right)$ depends smoothly on all its variables $J, \gamma_{1}$ and $\gamma_{2}$. Moreover, $\mathcal{G}$ is a quadratic structure on the trivial bundle $E\left(M, \mathbb{R}^{n}\right) / \mathbb{R}^{n} \times A^{1}\left(M, \mathbb{R}^{n}\right)$ and hence yields a metric on $E\left(M, \mathbb{R}^{n}\right) / \mathbb{R}^{n}$, denoted by $\mathcal{G}$, too.

We say that $F_{\mathbb{R}^{n}}$, a one-form on $E\left(M, \mathbb{R}^{n}\right) / \mathbb{R}^{n}$, admits an integral representation if there exists a smooth map

$$
\alpha: E\left(M, \mathbb{R}^{n}\right) \longrightarrow A^{1}\left(M, \mathbb{R}^{n}\right),
$$

called the kernel of $F_{\mathbb{R}^{n}}$, such that

$$
F_{\mathbb{R}^{n}}(\mathrm{~d} J)(\mathrm{d} L)=\int_{M} \alpha(\mathrm{d} J) \cdot \mathrm{d} L \mu(J)=\mathcal{G}(\mathrm{d} J)(\alpha(J), \mathrm{d} L)
$$

holds true for any choice of $\mathrm{d} J \in E\left(M, \mathbb{R}^{n}\right) / \mathbb{R}^{n}$ and $\mathrm{d} L \in C^{\infty}\left(M, \mathbb{R}^{n}\right) / \mathbb{R}^{n}$.

Definition 1 A constitutive law $F$ is a smooth one-form on $E\left(M, \mathbb{R}^{n}\right)$ with the following two properties
i.) $F=\mathrm{d}^{\star} F_{\mathbb{R}^{n}}$
ii.) $F_{\mathbb{R}^{n}}$ admits an integral representation (with kernel $\alpha$, say).

The kernel of a constitutive law is not unique at all. The following theorem provides us with a natural splitting of the kernel (and later allows us to extract from it a unique kernel of a specific kind). The proof can be found in [4]. 
Theorem 2 Let $\gamma \in A^{1}\left(M, \mathbb{R}^{n}\right)$ and $J \in E\left(M, \mathbb{R}^{n}\right)$. There exists a uniquely determined differential $\mathrm{d} \mathcal{H} \in C^{\infty}\left(M, \mathbb{R}^{n}\right) / \mathbb{R}^{n}$ called the exact part of $\gamma$ and a uniquely determined $\beta \in A^{1}\left(M, \mathbb{R}^{n}\right)$ such that

$$
\gamma=\mathrm{d} \mathcal{H}+\beta,
$$

where the exact part of $\beta$ vanishes. Both $\mathrm{d} \mathcal{H}$ and $\beta$ depend smoothly on $J$. If $\mathcal{H}\left(p_{0}\right)$ for some $p_{0} \in M$ is kept constant in $J$, then also $\mathcal{H}$ varies smoothly in $J$.

The effect of the splitting of kernels of constitutive laws is described in the following theorem, proved in [4] (cf. also [5]) :

Theorem 3 Let $F$ be any constitutive law. There exists a smooth map $\mathcal{H} \in$ $C^{\infty}\left(E\left(M, \mathbb{R}^{n}\right) / \mathbb{R}^{n}, C^{\infty}\left(M, \mathbb{R}^{n}\right)\right)$, such that for any $J \in E\left(M, \mathbb{R}^{n}\right)$ and any $L \in C^{\infty}\left(M, \mathbb{R}^{n}\right)$

$$
F(J)(L)=\int_{M} \mathrm{~d} \mathcal{H}(\mathrm{d} J) \cdot \mathrm{d} L \mu(J)=\mathcal{G}(\mathrm{d} J)(\mathrm{d} \mathcal{H}(\mathrm{d} J), \mathrm{d} L)
$$

The kernel $\mathrm{d} \mathcal{H}: E\left(M, \mathbb{R}^{n}\right) / \mathbb{R}^{n} \longrightarrow C^{\infty}\left(M, \mathbb{R}^{n}\right) / \mathbb{R}^{n} \subset A^{1}\left(M, \mathbb{R}^{n}\right)$ is uniquely determined by $F$, the map $\mathcal{H}$ can be chosen such that $(i d, \mathcal{H}) \in \Gamma\left(T E_{0}\left(M, \mathbb{R}^{n}\right)\right.$, . the latter choice is unique, too.

Since the constitutive law $F$ is determined by $\mathcal{H}$, we call this map a constitutive map. With any constitutive law the smooth two-tensor in (5) associated with $\gamma=\mathrm{d} \mathcal{H}(\mathrm{d} J)$ is referred to as stress tensor $T(J)$ at the configuration $J$.

\section{The Relation Between the Customary Notion of the Stress Tensor and the Constitutive Map}

To see that the setting in [17] is included in the treatment presented here, we assume that $\operatorname{dim} M=n$ and the work $F(J)(L)$ depends on the metric $m(J)$ and its derivative $\operatorname{Dm}(J)(L)$ at $J$ in the direction of $L$ rather than $J$ and $L$, themselves. (We may work at a fixed $J$.) Moreover let us suppose that $F$ admits for all its variables an integral representation of the form

$$
F(J)(L)=\int_{M} \mathcal{T}(J) \cdot \mathrm{D} m(J)(L) \mu(J),
$$

with a two-tensor $\mathcal{T}(J)$ as kernel. The dot-product in the integrand is defined by representing both $\mathcal{T}(J)$ and $\operatorname{D} m(J)(L)$, respectively as strong bundle maps $K_{1}(J)$ and $K_{2}(J)(L)$ of $T M$ via the metric $m(J)$ and then proceeding as in (6), i.e.

$$
\mathcal{T}(J) \cdot \operatorname{D} m(J)(L):=\operatorname{Tr} K_{1}(J) \cdot \Pi_{2}(J)(L)
$$

Using (8) and (9) the verification of the following is straightforward : 


$$
\begin{aligned}
F(J)(L) & =\int_{M} \operatorname{Tr} K_{1}(J) \cdot I_{2}(J)(L) \mu(J)=\int_{M} \operatorname{Tr} K_{1}(J) \cdot \tilde{A}(\mathrm{~d} L, \mathrm{~d} J) \mu(J) \\
& =\int_{M} \alpha(\mathrm{d} J) \cdot \mathrm{d} L \mu(J)=\int_{M} \mathrm{~d} \mathcal{H}(\mathrm{d} J) \cdot \mathrm{d} L \mu(J)
\end{aligned}
$$

with $\alpha(\mathrm{d} J):=\mathrm{d} J K_{1}(J)$, holding for all the variables of $\mathrm{F}$. In case $J$ is an equilibrium condition the tensors $\mathcal{T}(J)$ and $\frac{1}{2} \mathrm{D} m(J)(L)$ correspond to the stress tensor and to the deformation tensor respectively. The calculation in (10) shows, moreover, that to each smooth stress tensor assignment in the setting of [17] there is a constitutive map. (Nevertheless the two-tensors $T(J)$ and $\mathcal{T}(J)$ may still differ !) Reading (10) backwards shows that it suffices to work with $\mathcal{T}(J)$ instead of $\mathcal{H}(J)$. This difference however is obsolete with respect to the resulting force densities (cf. remark to Theorem 4 ).

\section{Force Densities Associated with Constitutive Laws}

The purpose of this section is to present how to associate with any constitutive law at any configuration some well defined force densities, one acting upon the whole body, and an other one acting upon the boundary only. Vice versa any given pair of force densities satisfying an integrability condition will be obtained via a suitable constitutive law.

Throughout this section $F$ is a constitutive law with a kernel $\alpha$. By the previous theorem we may assume that $\alpha\left(E\left(M, \mathbb{R}^{n}\right) / \mathbb{R}^{n}\right) \subset C^{\infty}\left(M, \mathbb{R}^{n}\right) / \mathbb{R}^{n}$. The following theorem shows how to associate the force densities mentioned above to any constitutive map ( $\mathrm{cf}$. Append. 2). The existence of a solution of the Neumann problem can found in [15]. Theorem 4 Every constitutive law $F \in A^{1}\left(E\left(M, \mathbb{R}^{n}\right), \mathbb{R}\right)$ admits a smooth con-
stitutive map

$$
\mathcal{H}: E\left(M, \mathbb{R}^{n}\right) / \mathbb{R}^{n} \longrightarrow C^{\infty}\left(M, \mathbb{R}^{n}\right)
$$

such that $F$ can be expressed as

$$
F(J)(L)=\int_{M}<\Delta(J) \mathcal{H}(\mathrm{d} J), L>\mu(J)+\int_{\partial M}<\mathrm{d} \mathcal{H}(\mathrm{d} J)(\mathbf{n}), L>i_{\mathbf{n}} \mu(J),
$$

for each $J \in E\left(M, \mathbb{R}^{n}\right)$ and each $L \in C^{\infty}\left(M, \mathbb{R}^{n}\right)$. For all $J \in E\left(M, \mathbb{R}^{n}\right)$ the map $\mathcal{H}$ defines the force densities $\Phi$ and $\varphi$ respectively by

$$
\Phi(\mathrm{d} J):=\Delta(J) \mathcal{H}(\mathrm{d} J)
$$

and

$$
\varphi(\mathrm{d} J):=\mathrm{d} \mathcal{H}(\mathrm{d} J)(\mathbf{n})
$$

which satisfy due to the fundamental properties of $F$, the equation 


$$
0=\int_{M} \Phi(\mathrm{d} J) \mu(J)+\int_{\partial M} \varphi(\mathrm{d} J) i_{\mathrm{n}} \mu(J)
$$

Given vice versa two smooth maps $\Phi \in C^{\infty}\left(E\left(M, \mathbb{R}^{n}\right) / \mathbb{R}^{n}, C^{\infty}\left(M, \mathbb{R}^{n}\right)\right)$ and $\varphi \in$ $C^{\infty}\left(E\left(M, \mathbb{R}^{n}\right) / \mathbb{R}^{n}, C^{\infty}\left(\partial M, \mathbb{R}^{n}\right)\right)$, for which the equation (13) holds as an integrability condition, then there exists also a smooth map $\mathcal{H} \in C^{\infty}\left(E\left(M, \mathbb{R}^{n}\right) / \mathbb{R}^{n}\right.$, $C^{\infty}\left(M, \mathbb{R}^{n}\right)$ ) satisfying (11) and (12) for which $\mathcal{H}(\mathrm{d} J)$ is uniquely determined up to a constant for each $J \in E\left(M, \mathbb{R}^{n}\right)$. Moreover $\mathcal{H}$ is a constitutive map for the constitutive law $F$ given by the force densities via the formula

$$
F(J)(L)=\int_{M}<\Phi(\mathrm{d} J), L>\mu(J)+\int_{\partial M}<\varphi(\mathrm{d} J), L>i_{\mathbf{n}} \mu(J),
$$

holding for all $J \in E\left(M, \mathbb{R}^{n}\right)$ and for all $L \in C^{\infty}\left(M, \mathbb{R}^{n}\right)$. In case $\partial M=\emptyset$ then (12) and also the second terms on the right hand sides of both (19) and (14) disappear.

Remark. This theorem shows to us that our notion of constitutive laws (based on translational invariance) is equivalent with the $\mathbb{R}^{n}$-valued solution of Neumann problems formulated on $M$ and hence is equivalent with a pair of force densities satisfying the integrability condition (13), which tells us that the resulting force density to the center of mass vanishes. (13) is obviously the analog of (2). Moreover, by Theorem 3 a general kernel $\alpha$ and its exact part both determine the same force densities!

\section{The Interplay Between Constitutive Laws of Boundary and Body}

The deformable media forming the inside of the body and the boundary respectively may differ and each separate material hence has to be described on one hand by different constitutive laws, as we have done in the previous sections. On the other hand both materials together form one body and should be describable by only one constitutive law holding for the whole body. Since constitutive laws behave additively, the comparison between the two procedures allows us to decode the influence of the whole body to the constitutive properties of the boundary material.

Let $F$ be the constitutive law of the deformable medium forming the whole body. According to Theorem 4 the one-form $F$ is determined by a smooth constitutive map $\mathcal{H}$. It affects the quality of the boundary material: $\mathcal{H}$ yields according to Theorem 4 force densities $\Phi \in C^{\propto}\left(E\left(M, \mathbb{R}^{n}\right) / \mathbb{R}^{n}, C^{\propto}\left(M, \mathbb{R}^{n}\right)\right)$ and $\varphi \in C^{\infty}\left(E\left(M, \mathbb{R}^{n}\right) / \mathbb{R}^{n}, C^{\infty}\left(\partial M, \mathbb{R}^{n}\right)\right)$.

The force density acting on $\partial M$, is defined by 


$$
\varphi(\mathrm{d} J)=\mathrm{d} \mathcal{H}(\mathrm{d} J)(\mathbf{n}), \quad \forall \mathrm{d} J \in E\left(M, \mathbb{R}^{n}\right) / \mathbb{R}^{n} .
$$

Having the integrability condition (13) for $\Delta(J \mid \partial M)$ in mind, we split this force density $\varphi$ into

$$
\varphi(\mathrm{d} J)=\varphi_{\mathbb{R}^{n}}(\mathrm{~d} J)+\psi(\mathrm{d} J), \quad \forall \mathrm{d} J \in E\left(M, \mathbb{R}^{n}\right) / \mathbb{R}^{n},
$$

where $\varphi_{\mathbb{R}^{n}}(\mathrm{~d} J)$ is characterized for each $\mathrm{d} J \in E\left(M, \mathbb{R}^{n}\right) / \mathbb{R}^{n}$ by the equation

$$
\int_{\partial M} \varphi \mathbb{R}^{n}(\mathrm{~d} J) i_{\mathbf{n}} \mu(J)=0 .
$$

The map $\psi \in C^{\infty}\left(E\left(M, \mathbb{R}^{n}\right) / \mathbb{R}^{n}, \mathbb{R}^{n}\right)$ is $L_{2}$-orthogonal to $\varphi_{\mathbb{R}^{n}}$. According to Theorem 4 the condition (15) allows us to choose some map

$$
\eta \in C^{\infty}\left(E\left(M, \mathbb{R}^{n}\right) / \mathbb{R}^{n}, C^{\infty}\left(\partial M, \mathbb{R}^{n}\right)\right),
$$

such that for all $\mathrm{d} J \in E\left(M, \mathbb{R}^{n}\right) / \mathbb{R}^{n}$ the equation

$$
\varphi_{\mathbb{R}^{n}}(\mathrm{~d} J)=\Delta(J \mid \partial M) h(\mathrm{~d} J)
$$

holds true. With these data we easily verify the following :

Theorem 5 Any smoothly deformable medium is characterized by a constitutive map $\mathcal{H} \in C^{\infty}\left(E\left(M, \mathbb{R}^{n}\right) / \mathbb{R}^{n}, C^{\infty}\left(M, \mathbb{R}^{n}\right)\right)$, that determines itself two smooth maps $\eta \in C^{\infty}\left(E\left(M, \mathbb{R}^{n}\right) / \mathbb{R}^{n}, C^{\infty}\left(\partial M, \mathbb{R}^{n}\right)\right)$ and $\psi \in C^{\infty}\left(E\left(M, \mathbb{R}^{n}\right), \mathbb{R}^{n}\right)$, which are linked to $\mathcal{H}$ by the boundary condition

$$
\mathrm{d} \mathcal{H}(\mathrm{d} J)(\mathbf{n})=\Delta(J \mid \partial M) h(\mathrm{~d} J)+\psi(\mathrm{d} J)
$$

for each $J \in E\left(M, \mathbb{R}^{n}\right)$. The differential $\mathrm{d} h$ and $\psi \in C^{\infty}\left(E\left(M, \mathbb{R}^{n}\right) / \mathbb{R}^{n}, \mathbb{R}^{n}\right)$ are unique. For each $J \in E\left(M, \mathbb{R}^{n}\right)$ the map $\mathcal{H}$ satisfies

$$
0=\int_{M} \Delta(J) \mathcal{H}(\mathrm{d} J) \mu(J)+\int_{\partial M} \psi(\mathrm{d} J) i_{\mathrm{n}} \mu(J) .
$$

The constitutive law on $E\left(M, \mathbb{R}^{n}\right)$ describing the constitutive properties of the materials forming body together with its boundary is determined by $\mathcal{H}$, which yields $\eta$ and $\psi$ and thus is given via the formula

$$
\begin{aligned}
F(J)(L) & =\int_{M}<\Delta(J) \mathcal{H}(\mathrm{d} J), L>\mu(J) \\
& +\int_{\partial M}<\Delta(J \mid \partial M) h(J \mid \partial M)+\psi(\mathrm{d} J), L>i_{\mathbf{n}} \mu(J)
\end{aligned}
$$

valid for all variables' of $F$. The work of any distortion $l \in C^{\infty}\left(\partial M, \mathbb{R}^{n}\right)$ of the deformable material forming the boundary regarded as being detached from the body, is for any $J \in E\left(M, \mathbb{R}^{n}\right)$ given by the constitutive law

$$
F_{\partial M}(\mathrm{~d} J)(l)=\int_{\partial M}<\Delta(J \mid \partial M) \boldsymbol{\eta}_{\partial}(\mathrm{d} J), l>i_{\mathbf{n}} \mu(J)
$$


for some constitutive map $h_{\partial} \in C^{\infty}\left(O_{\partial} / \mathbb{R}^{n}, C^{\infty}\left(\partial M, \mathbb{R}^{n}\right)\right)$. Hence the difference $\eta-h_{\partial}$ and $\psi$ describe how the constitutive properties of the material forming the boundary of the body are affected by the fact that this material is incorporated into the material forming the whole body. (Any $h_{\partial}(\mathrm{d} J)$ can be harmonically extended to all of $M$ ).

\section{A General Decomposition of Constitutive Laws}

In this section we will exhibit decompositions of $\mathrm{d} \mathcal{H}$ and $\mathrm{d} \not$ as induced by (16). Both splittings are based on the examples in Append. 3. We need the maps $\mathcal{V}, \mathcal{A}$ and $N$, describing for each configuration the volume, the area and the shape respectively (cf. (iii) in Append. 3). In particular we will show that $\mathrm{D} \mathcal{V}$ and $R^{\star} \mathrm{D} \mathcal{A}$ (cf. Sect. 2 for $\mathrm{R}$ ) multiplied with appropriate $\mathbb{R}$-valued functions are part of any constitutive law $F$ defined on $E\left(M, \mathbb{R}^{n}\right)$. Again let $j:=J \mid \partial M$.

To obtain the desired decomposition of a given constitutive law we broaden our scope a little and, first of all, introduce the Hilbert space $A_{j}$ consisting of all maps $\gamma_{1}, \gamma_{2}: T \partial M \longrightarrow \mathbb{R}$ linear on the fibers of $T \partial M$ for which the right hand side of

$$
G(\mathrm{~d} j)\left(\gamma_{1}, \gamma_{2}\right):=\int_{\partial M} \gamma_{1} \cdot \gamma_{2} \mu(j)
$$

exists. Clearly $\mathrm{d} \eta_{\mathbf{n}}(\mathrm{d} J)$ (defined in Append. 3 ), $\mathrm{d} j$ and $\mathrm{d} N(j)$ all belong to $A_{j}$ and are generically linearly independent. In the special case of $j(\partial M)$ being a $(n-1)$ sphere in $\mathbb{R}^{n}$ however, $N(j), j$ and $\eta_{\mathbf{n}}(\mathrm{d} J)$ are not linearly independent. The set $O_{3}$ of all $J \in E\left(M, \mathbb{R}^{n}\right)$ for which these three differentials are linearly independent forms a dense open set in $E\left(M, \mathbb{R}^{n}\right)$.

For each $J \in O_{3}$ we split the differential of $h(\mathrm{~d} J)$ into components along the span of the three mentioned differentials and a component perpendicular to it. The next step is to define maps $\left(\eta_{\mathbf{n}}(\mathrm{d} J)\right)_{M}, j_{M}$ and $N(j)_{M}$ associated with $\eta_{\mathbf{n}}(\mathrm{d} J), j$ and $N(j)$ respectively. This is done by solving the following Visik problem (cf. $[15])$ : Let $f \in C^{\infty}\left(\partial M, \mathbb{R}^{n}\right)$ be given. We define $f_{M}(J) \in C^{\infty}\left(M, \mathbb{R}^{n}\right)$ by

$$
\Delta(J) f_{M}(J)=0 \text { and } \mathrm{d} f_{M}(J)(\mathbf{n})-\Delta(j) f=0
$$

for any $J \in E\left(M, \mathbb{R}^{n}\right)$. In particular $\eta_{\mathrm{n}}$ satisfies

$$
\mathrm{d}\left(\eta_{\mathbf{n}}(\mathrm{d} J)\right)_{M}=\mathrm{d} J, \quad \forall \in E\left(M, \mathbb{R}^{n}\right) .
$$

$f_{M}$ depends smoothly on $J$. The above mentioned decomposition of $\mathrm{d} \mathcal{H}$ is then presented in the following theorem partly based on Theorem 5 :

Theorem 6 Let $F$ be a constitutive law on $E\left(M, \mathbb{R}^{n}\right)$. Then any of its constitutive maps $\mathcal{H} \in C^{\infty}\left(E\left(M, \mathbb{R}^{n}\right) / \mathbb{R}^{n}, C^{\infty}\left(M, \mathbb{R}^{n}\right)\right)$ determines uniquely three smooth functions 


$$
a_{1}, a_{2}, a_{3}: O_{3} / \mathbb{R}^{n} \subset E\left(M, \mathbb{R}^{n}\right) / \mathbb{R}^{n} \longrightarrow \mathbb{R}
$$

and the smooth maps

$$
\begin{gathered}
\not, \not_{2}: O_{3} / \mathbb{R}^{n} \subset E\left(M, \mathbb{R}^{n}\right) / \mathbb{R}^{n} \longrightarrow C^{\infty}\left(\partial M, \mathbb{R}^{n}\right), \\
\psi: O_{3} \subset E\left(M, \mathbb{R}^{n}\right) \longrightarrow \mathbb{R}^{n}
\end{gathered}
$$

linked to $\mathcal{H}$ by

$$
\mathrm{d} \mathcal{H}(\mathrm{d} J)(\mathbf{n})=\Delta(j) h(\mathrm{~d} J)+\psi(\mathrm{d} J),
$$

such that the following splitting holds for any $J \in O_{3} \subset E\left(M, \mathbb{R}^{n}\right)$

$$
\mathrm{d} h(\mathrm{~d} J)=a_{1}(\mathrm{~d} J) \cdot \mathrm{d} \eta_{\mathbf{n}}(\mathrm{d} J)+a_{2}(\mathrm{~d} J) \mathrm{d} j+a_{3}(\mathrm{~d} J) \cdot \mathrm{d} N(j)+\mathrm{d} \eta_{2}(\mathrm{~d} J)
$$

with $j:=J / \partial M$. The differential $\mathrm{d} h_{2}(\mathrm{~d} J)$ is with respect to $G(\mathrm{~d} j)$ orthogonal to the span of $\mathrm{d} \eta_{\mathrm{n}}(\mathrm{d} J), \mathrm{d} j$ and $\mathrm{d} N(j)$. The differential $\mathrm{d} \mathcal{H}(\mathrm{d} J)$ decomposes for each $J \in \mathrm{O}_{3}$ accordingly into

$$
\mathrm{d} \mathcal{H}(\mathrm{d} J)=a_{1}(\mathrm{~d} J) \cdot \mathrm{d} J+a_{2}(\mathrm{~d} J) \cdot \mathrm{d} j_{M}+a_{3}(\mathrm{~d} J) \cdot \mathrm{d} N_{M}(\mathrm{~d} J)+\mathrm{d} \mathcal{H}_{2}(\mathrm{~d} J),
$$

where $\mathrm{d} \mathcal{H}_{2}(\mathrm{~d} J)$ is such that (19) holds. The splittings (18) and (19) are valid for the respective maps if the representatives of the differentials are chosen from $E_{0}\left(M, \mathbb{R}^{n}\right)$.

\section{Two Simple Bubble Models}

Let us illustrate the meaning of the coefficients $a_{1}$ and $a_{2}$ in (17) by a simple bubble model. ( $\mathrm{d} I$ and $\mathrm{d} i$ as arguments will be omitted.) Let $\operatorname{dim} M=3$ and let us think of $M$ as a bubble and of $\partial M$ as the middle surface of a very thin shell bounding the bubble. Moreover we assume $a_{3}=0$ and $\mathcal{H}_{2}=0$ as well and suppose that there is an equilibrium configuration $I \in E\left(M, \mathbb{R}^{n}\right)$, i.e. to say we suppose that $F(I)=0$. Since $\partial M$ represents two bounding surfaces, (18) turns into

$$
0=a_{1} \cdot \mathrm{d} \eta_{\mathbf{n}}+2 \cdot a_{2} \mathrm{~d} i .
$$

Using the respective formulas in Append. 3, we find

$$
a_{1}+2 \cdot a_{2} \cdot H=0,
$$

with $\mathrm{H}$ the mean curvature of $i(M) \subset \mathbb{R}^{3}$. Thus we observe that $\mathrm{H}$ is a constant map on $M$ and deduce from the classical bubble models, therefore, that $a_{1}$ and $a_{2}$ can be interpreted as an internal pressure and as a capillarity respectively.

If the quality of the deformable medium depends in addition on the shape, i.e. if $a_{3} \neq 0$ (but still $\mathcal{H}_{2}=0$ ) at the equilibrium configuration $I$ (which might be an immersion only), we find due to (18) and (A5) the following equation

$$
0=a_{1}+2 a_{2} \cdot H+2 a_{3} \cdot H^{2}-2 a_{3} \kappa,
$$


with $\kappa$ the Gaussian curvature. By integrating (20) and by using the theorem of Gauss-Bonnet we deduce

$$
0=a_{1}+2 a_{2} \cdot H+2 a_{3} \cdot H^{2}-4 \pi a_{3} \frac{\chi(\partial M)}{A},
$$

with $A$ the area of $\partial M$. Equation (21) in turn yields

$$
\chi(\partial M)=\frac{1}{2 \pi} \cdot \kappa \cdot A .
$$

One now easily shows that $\mathrm{H}$ is constant and that in this case $\partial M$ cannot be a sphere. If $I$ exists at all, $i(\partial M) \subset \mathbb{R}^{3}$ has to be an immersed torus as seen by a theorem of Efimov (cf. [2]).

\section{The Rotational Symmetry}

From the two symmetries described in Sect. 1 we have used so far the translational symmetry only. It is clear, however, that internal physical processes are also invariant under the rotational symmetry. In this section we will show what additional property any constitutive map inherits from this symmetry.

Invariance under $\mathrm{SO}(n)$ of a given constitutive law $F$ with $\mathcal{H}$ as a constitutive map yields

$$
F(g \circ J)(g \circ L)=F(J)(L)
$$

for all the variables of $F$ and for any $g \in \mathrm{SO}(n)$. Thus the constitutive map satisfies

$$
\int_{M} \mathrm{~d} \mathcal{H}(g \circ \mathrm{d} J) \cdot \mathrm{d}(g \circ L) \mu(g \circ J)=\int_{M} \mathrm{~d} \mathcal{H}(\mathrm{d} J) \cdot \mathrm{d} L \mu(J)
$$

and hence

$$
\int_{M} \mathrm{~d}\left(g^{-1} \circ \mathcal{H}(g \circ \mathrm{d} J)-\mathcal{H}(\mathrm{d} J)\right) \cdot \mathrm{d} L \mu(J)=0
$$

for all $g \in \mathrm{SO}(n)$, for all $J \in E\left(M, \mathbb{R}^{n}\right)$ and for all $L \in C^{\infty}\left(M, \mathbb{R}^{n}\right)$. From the last equation and from the general procedure of representing differentials via embeddings we read off :

Proposition 7 Given a constitutive law $F$ with constitutive map

$$
\mathcal{H} \in C^{\infty}\left(E\left(M, \mathbb{R}^{n}\right) / \mathbb{R}^{n}, C^{\infty}\left(M, \mathbb{R}^{n}\right)\right),
$$

then $F$ is invariant under $\mathrm{SO}(n)$ iff $\mathcal{H}$ is equivariant, i.e. iff

$$
g^{-1} \circ \mathrm{d} \mathcal{H}(g \circ \mathrm{d} J)=\mathrm{d} \mathcal{H}(\mathrm{d} J), \quad \forall G \in \mathrm{SO}(n) .
$$

The infinitesimal version of (22) is

$$
\mathrm{dD} \mathcal{H}(\mathrm{d} J)(c)=c \mathrm{~d} \mathcal{H}(\mathrm{d} J), \quad \forall c \in s o(n) .
$$


The validity of (22) implies in particular the following identity:

$$
A(\mathrm{~d} \mathcal{H}(g \circ \mathrm{d} J), g \circ \mathrm{d} J)=A(\mathrm{~d} \mathcal{H}(\mathrm{d} J), \mathrm{d} J)
$$

holding for all $g \in \mathrm{SO}(n)$ and for all $J \in E\left(M, \mathbb{R}^{n}\right)$. The stress tensor $T(J)$ determined by $\mathcal{H}$ is hence invariant under $\mathrm{SO}(n)$ for any $J \in E\left(M, \mathbb{R}^{n}\right)$.

If $F$ factors to $T_{J} E\left(M, \mathbb{R}^{n}\right) / s o(n) \cdot J$, and thus $F(J)(c J)=0$ for all $c \in \operatorname{so}(n)$ then

$$
\int_{M}<c \phi(\mathrm{d} J), J>\mu(J)+\int_{\partial M}<c \varphi(\mathrm{d} J), J>i_{\mathbf{n}} \mu(J)=0
$$

holds. This is the analog to (2). If $\operatorname{dim} M=3$, then $E\left(M, \mathbb{R}^{n}\right) / \mathrm{SO}(n)$ plays a fundamental role in continuum mechanics (cf. [19]).

\section{A Generalization of the Notion of a Constitutive Law and the Introduction of a Dynamics}

In this section we introduce a dynamics in case $\partial M=\emptyset$. All constructions could be performed for any codimension of the body in $\mathbb{R}^{n}$. However, due to simplicity we require $\operatorname{dim} M=n-1$. Here we follow closely [7]. First of all we extend the notion of a constitutive law. In doing so we consider special one-forms on $T E\left(M, \mathbb{R}^{n}\right)$ namely those of the type

$$
\hat{F}_{\mathbb{R}^{n}}: T^{2}\left(E\left(M, \mathbb{R}^{n}\right) / \mathbb{R}^{n}\right) \longrightarrow \mathbb{R}
$$

satisfying

$$
\hat{F}_{\mathbb{R}^{n}}(\mathrm{~d} j, \mathrm{~d} k)(\mathrm{d} l, \mathrm{~d} s)=\hat{F}_{\mathbb{R}^{n}}(\mathrm{~d} j, \mathrm{~d} k)(\mathrm{d} l, 0),
$$

for any $\mathrm{d} j \in E\left(M, \mathbb{R}^{n}\right) / \mathbb{R}^{n}$ and for any choice of $\mathrm{d} k, \mathrm{~d} l, \mathrm{~d} s \in C^{\infty}\left(M, \mathbb{R}^{n}\right) / \mathbb{R}^{n}$.

A constitutive law (depending not only on the configurations but also on the velocities $)$ is then a one-form $F$ on $T E\left(M, \mathbb{R}^{n}\right)$ satisfying for all $\mathrm{d} j \in E\left(M, \mathbb{R}^{n}\right) / \mathbb{R}^{n}$ and all $\mathrm{d} k, \mathrm{~d} l \in C^{\infty}\left(M, \mathbb{R}^{n}\right) / \mathbb{R}^{n}$

$$
F(j, k)(l)=\hat{F}_{\mathbb{R}^{n}}(\mathrm{~d} j, \mathrm{~d} k)(\mathrm{d} l, 0),
$$

and admitting an integral representation. The constitutive map $\mathcal{H}$ depends hence on configurations and velocities!

Next we will define motions subjected to a constitutive law by using d'Alembert's principle:

A motion on $E\left(M, \mathbb{R}^{n}\right)$ in our model is described by a smooth curve of embeddings

$$
\sigma:(-\lambda, \lambda) \longrightarrow E\left(M, \mathbb{R}^{n}\right),
$$

for some positive real $\lambda$. This motion is subjected to the constitutive law $F$, if 


$$
F(\dot{\sigma}(t))(\sigma(t), h)=\mathcal{B}(\sigma(t))(\ddot{\sigma}(t), h), \quad \forall h \in T_{\sigma(t)} E\left(M, \mathbb{R}^{n}\right)
$$

is satisfied for all $t \in(-\lambda, \lambda)$ with $\mathcal{B}$ as in Append. 4. Hence

$$
\int_{M}<\Phi(\sigma(t), \dot{\sigma}(t)), h>\mu(\sigma(t))=\int_{M} \rho(\sigma(t))<\ddot{\sigma}(t), h>\mu(\sigma(t))
$$

has to hold for $h$ and for all $t$, mentioned above (cf. [13],[20],[22]). Here $\Phi$ is the force density determined by $F$. This implies Newton's third law of motion on the density level:

$$
\rho(\sigma(t)) \ddot{\sigma}(t)=\Phi(\sigma(t), \dot{\sigma}(t))=\Delta((\sigma(t)) \mathcal{H}(\mathrm{d} \sigma(t), \mathrm{d} \dot{\sigma}(t)), \quad \forall t \in(-\lambda, \lambda) .
$$

Clearly $\sigma$ parameterizes a straight line segment, i.e. a geodesic of $\mathcal{B}$, iff: $\Phi(\sigma(t), \dot{\sigma}(t))=0$ for all $t \in(-\lambda, \lambda)$.

Let $\sigma$ be a motion satisfying (23). We rewrite $\sigma(t)$ according to the principal bundle structure of $E\left(M, \mathbb{R}^{n}\right)$ (cf. [6]), by proceeding as follows: At first we note that $\dot{\sigma}(t)$ admits in $\mathbb{R}^{n}$ the pointwise splitting

$$
\dot{\sigma}(t)=\mathrm{d} \sigma(t) Z(t)+\dot{\sigma}(t)^{\perp}
$$

and easily verify

$$
\begin{aligned}
\mathrm{d} \dot{\sigma}(t) Z(t) & =\mathrm{d}(\mathrm{d} \sigma(t) Z(t)) Z(t)+\mathrm{d} \dot{\sigma}(t)^{\perp} Z(t) \\
& =\sigma(t) \nabla(\sigma(t)) Z(t) Z(t)+S(\sigma(t))(Z(t), Z(t)) \\
& +\mathrm{d} \sigma(t) W(\sigma(t)) Z(t)+\left(\mathrm{d} \dot{\sigma}(t)^{\perp} Z(t)\right)^{\perp}
\end{aligned}
$$

where " $\perp$ " denotes the pointwise formed component normal to $\sigma(t)(M)$. Moreover we have for each $t \in(-\lambda, \lambda)$ and for some well-defined functions $\epsilon(\sigma(t), \dot{\sigma}(t)) \in$ $C^{\infty}(M, \mathbb{R})$ and $K_{F}(\sigma(t), \dot{\sigma}(t)) \in C^{\infty}(M, \mathbb{R})$

$$
\dot{\sigma}(t)^{\perp}=\epsilon(\sigma(t), \dot{\sigma}(t)) N(\sigma(t))
$$

and

$$
\Phi(\sigma(t), \dot{\sigma}(t))^{\perp}=K_{F}(\sigma(t), \dot{\sigma}(t)) N(\sigma(t))
$$

respectively. Using the formula

$$
\begin{aligned}
\dot{N}(\sigma(t)) & =\mathrm{D} N(\sigma(t))(\dot{\sigma}(t)) \\
& =\mathrm{d} \sigma(t)\left(W(\sigma(t)) Z(t)-\operatorname{grad}_{\sigma(t)} \epsilon(\sigma(t), \dot{\sigma}(t))\right),
\end{aligned}
$$

we find the following system for a motion on $E\left(M, \mathbb{R}^{n}\right)$ subjected to a constitutive law:

$$
\begin{aligned}
& \nabla(\sigma(t))_{Z(t)} Z(t)+\dot{Z}(t)+2 \epsilon(\sigma(t), \dot{\sigma}(t)) W(\sigma(t)) Z(t) \\
& -\epsilon(\sigma(t), \dot{\sigma}(t)) \operatorname{grad}_{\sigma(t)} \epsilon(\sigma(t), \dot{\sigma}(t)) \\
& =\rho^{-1}(\sigma(t)) Y_{F}(\sigma(t), \dot{\sigma}(t))
\end{aligned}
$$

and 


$$
\begin{aligned}
\dot{\epsilon}(\sigma(t), \dot{\sigma}(t)) & =\rho^{-1}(\sigma(t)) \Pi_{F}(\sigma(t), \dot{\sigma}(t)) \\
& +\mathbf{f}(\sigma(t))(Z(t), Z(t))-\mathrm{d} \epsilon(\sigma(t), \dot{\sigma}(t)) Z(t),
\end{aligned}
$$

with $Y_{F}(\sigma(t), \dot{\sigma}(t)) \in \Gamma T M$ such that $\mathrm{d} J Y_{F}(\sigma(t), \dot{\sigma}(t))=\Phi(\sigma(t), \dot{\sigma}(t))^{\top}$ for all $t \in(-\lambda, \lambda)$. Here $\mathbf{f}(\sigma(t))$ denotes the second fundamental form of $\sigma(t)$ (cf. Append. $1)$ and "T" denotes the pointwise formed component tangential to $\sigma(t)(M)$.

Remark. Equation (24) describes a straight line segment in $E\left(M, \mathbb{R}^{n}\right)$ iff both $Y_{F}(\sigma(t), \dot{\sigma}(t))=0$ and $K_{F}(\sigma(t), \dot{\sigma}(t))=0$ hold for all $t \in(-\lambda, \lambda)$. In this case the equations (24) read as

$$
\begin{gathered}
\nabla(\sigma(t))_{Z(t)} Z(t)+\dot{Z}(t)+2 \epsilon(\sigma(t), \dot{\sigma}(t)) W(\sigma(t)) Z(t) \\
-\epsilon(\sigma(t), \dot{\sigma}(t)) \operatorname{grad}_{\sigma(t)} \epsilon(\sigma(t), \dot{\sigma}(t))=0 \\
\dot{\epsilon}(\sigma(t), \dot{\sigma}(t))=\mathbf{f}(\sigma(t))(Z(t), Z(t))-\mathrm{d} \epsilon(\sigma(t), \dot{\sigma}(t)) Z(t) .
\end{gathered}
$$

Otherwise (24) describes the deviation from geodesics.

\section{A Navier-Stokes Type of Equation}

Again $M, F$, and $\mathcal{H}$ are as in the previous section. The introduction of a structural viscosity coefficient relies on a mathematical observation : If we write any $k \in$ $C^{\infty}\left(M, \mathbb{R}^{n}\right)$ in the form

$$
k=\mathrm{d} j X(k, j)+\theta(k, j) \cdot N(j),
$$

where $X(k, j) \in \Gamma T M$ and $\theta(k, j) \in C^{\infty}\left(M, \mathbb{R}^{n}\right)$ both are uniquely determined by $k$, then we observe that $X^{0}(k, j)$, the divergence-free part of $X(k, j)$, is uniquely determined by $\mathrm{d} k$. This motivates us to introduce a structural viscosity $\nu(\mathrm{d} j, \mathrm{~d} k) \in$ $C^{\infty}(M, \mathbb{R})$ via

$$
X(\mathcal{H}(\mathrm{d} j, \mathrm{~d} k), j)=\nu(\mathrm{d} j, \mathrm{~d} k) X^{0}(k, j)+\hat{X}(\mathcal{H}(\mathrm{d} j, \mathrm{~d} k) ; j)
$$

The second term in (26) is such that this equation holds. The following theorem describes a generalization of the Navier-Stokes equation (cf. [7]):

Theorem 8 In absence of external force densities, the general equations of motion on $E\left(M, \mathbb{R}^{n}\right)$ of a deformable medium subjected to a constitutive law $F$ are given by

$$
\begin{aligned}
& \nabla(\sigma(t))_{Z(t)} Z(t)+\dot{Z}(t)+2 \cdot \epsilon(\sigma(t), \dot{\sigma}(t)) W(\sigma(t)) Z(t) \\
& -\epsilon(\sigma(T), \dot{\sigma}(T)) \operatorname{grad}_{\sigma(t)} \epsilon(\sigma(t), \dot{\sigma}(t)) \\
& =\rho^{-1}(\sigma(t))\left[\operatorname{grad}_{\sigma(t)} \tau_{i n t}(\sigma(t), \dot{\sigma}(t))\right. \\
& +\Delta(\sigma(t))\left(\nu(\mathrm{d} \sigma(t), \mathrm{d} \dot{\sigma}(t)) Z^{0}(t)+\hat{X}(\mathcal{H}(\mathrm{d} \sigma(t), \mathrm{d} \dot{\sigma}(t)), \sigma(t))\right) \\
& -2 \cdot W(\sigma(t)) \operatorname{grad}_{\sigma(t)} \theta(\mathcal{H}(\mathrm{d} \sigma(t), \mathrm{d} \dot{\sigma}(t)), \sigma(t)) \\
& +\left(W(\sigma(t))^{2}\left(\nu(\mathrm{d} \sigma(t), \mathrm{d} \dot{\sigma}(t)) Z^{0}(t)+\hat{X}(\mathcal{H}(\sigma(t), \dot{\sigma}(t)), \sigma(t))\right)\right. \\
& \left.-\theta(\mathcal{H}(\mathrm{d} \sigma(t), \mathrm{d} \dot{\sigma}(t)), \sigma(t)) \cdot \operatorname{grad}_{\sigma(t)} \mathcal{H}(\sigma(t))\right]
\end{aligned}
$$




$$
\begin{aligned}
\dot{\epsilon}(\sigma(t), \dot{\sigma}(t)) & =\rho^{-1}(\sigma(t))\left[-\tau_{\text {int }}(\mathrm{d} \sigma(t), \mathrm{d} \dot{\sigma}(t)) H(\sigma(t))\right. \\
& -\mathrm{d} H(\sigma(t))\left(\nu(\mathrm{d} \sigma(t), \mathrm{d} \dot{\sigma}(t)) Z^{0}(t)+\hat{X}(\mathcal{H}(\mathrm{d} \sigma(t), \mathrm{d} \dot{\sigma}(t)), \sigma(t))\right) \\
& +2 \cdot \operatorname{div}_{\sigma(t)} \nu(\mathrm{d} \sigma(t), \mathrm{d} \dot{\sigma}(t)) W(\sigma(t)) Z^{0}(t) \\
& +2 \cdot \operatorname{div}_{\sigma(t)} W(\sigma(t)) \hat{X}(\mathcal{H}(\mathrm{d} \sigma(t), \mathrm{d} \dot{\sigma}(t)), \sigma(t)) \\
& +\theta(\mathcal{H}(\mathrm{d} \sigma(t), \mathrm{d} \dot{\sigma}(t)), \sigma(t)) \cdot \operatorname{Tr} W(\sigma(t))^{2} \\
& +\Delta(\sigma(t)) \theta(\mathcal{H}(\mathrm{d} \sigma(t), \mathrm{d} \dot{\sigma}(t)), \sigma(t))] \\
& +\mathbf{f}(\sigma(t))(Z(t), Z(t))-\mathrm{d} \epsilon(\sigma(t), \dot{\sigma}(t)) Z(t),
\end{aligned}
$$

where $Z^{0}(t):=X^{-0}(\dot{\sigma}(t), \sigma(t))$. The motion of a deformable medium along a fixed surface $i(M) \subset \mathbb{R}^{n}$ is given by

$$
\begin{aligned}
\nabla(i)_{V(t)} V(t)+\dot{V}(t) & =\rho^{-1}\left(\sigma(t) \circ g(t)^{-1}\right)\left[\operatorname{grad}_{i} \tau_{\text {int }}(\sigma(t), V(t))\right. \\
& +\Delta(i)\left(\nu(\sigma(t), V(t)) V^{0}(t)+\hat{V}(\mathcal{H}(\sigma(t), V(t)), V(t))\right) \\
& -2 \cdot W(i) \operatorname{grad}_{i} \theta(\mathcal{H}(\sigma(t), V(t)), V(t)) \\
& +(W(i))^{2}\left(\nu(\sigma(t), V(t)) V^{0}(t)+\hat{V}(\mathcal{H}(\sigma(t), V(t)), V(t))\right. \\
& \left.-\theta(\mathcal{H}(\sigma(t), V(t)), V(t)) \cdot \operatorname{grad}_{i} H(i)\right] \\
0= & \rho^{-1}\left(\sigma(t) \circ g(t)^{-1}\right)\left[-\tau_{i n t}(\sigma(t), V(t)) \cdot H(i)\right. \\
- & \mathrm{d} H(i)\left(\nu(\sigma(t), V(t)) V^{0}(t)+\hat{V}(\mathcal{H}(\sigma(t), V(t)), V(t))\right) \\
+ & 2 \cdot\left(\operatorname{div}_{i} \nu(\sigma(t), V(t)) W(i) V^{0}(t)\right. \\
+ & \left.\operatorname{div}_{i} W(i) \hat{V}(\mathcal{H}(\sigma(t), V(t)), V(t))\right) \\
+ & \theta(\mathcal{H}(\sigma(t), V(t)), V(t)) \cdot \operatorname{Tr} W(i)^{2} \\
+ & \Delta(\sigma(t)) \theta(\mathcal{H}(\sigma(t), V(t)), V(t))] \\
+ & \mathbf{f}(i)(V(t), V(t)),
\end{aligned}
$$

where $\sigma(t)=i \circ g(t)$ with $g(t) \in \operatorname{Diff} M$. The vector fields $V(t), V^{0}(t)$ and $\hat{V}(\mathcal{H}(\sigma(t), V(t)), V(t))$ are respectively the push-forwards of $Z(t), Z^{0}(t)$ and $\hat{X}(\mathcal{H}(\mathrm{d} \sigma(t), \mathrm{d} \dot{\sigma}(t)), \sigma(t))$ by $g(t)$. The map $\tau_{\text {int }} \in C^{\infty}\left(M, \mathbb{R}^{n}\right)$ is given by decomposing the tangential component of the force density via Hodge's method into a gradient (of $\tau_{\text {int }}$ ) and a divergence-free part.

As to whether this Navier-Stokes type of equation is invariant under the substitution $t \mapsto-t$ depends on the map $\nu$.

As an application of the above approach and of the remark to Theorem 4 let us consider the following special case, determined by the form

$$
\alpha(\mathrm{d} j, \mathrm{~d} k)=-\tau_{\text {int }}(\mathrm{d} j, \mathrm{~d} k) \cdot \mathrm{d} j+\nu \cdot \mathrm{d} j \cdot L_{V^{0}(k, j)},
$$

with a constant $\nu \in \mathbb{R}$ and where $L_{V^{0}(k, j)}$ denotes the Lie derivative on $\Gamma T M$ in the direction $V^{0}(k, j)$. In the incompressible case, i.e. for $\operatorname{div}_{i} V(t)=0$, the motion along the fixed manifold $i(M) \subset \mathbb{R}^{n}$ is governed by 


$$
\begin{aligned}
& \rho^{-1}(i) \cdot \operatorname{grad}_{i} \tau_{\text {int }}(\sigma(t), V(t))+\nu \cdot \Delta(i) V(t)-\nu \cdot \operatorname{Ric}(i) V(t) \\
& =\nabla(i)_{V(t)} V(t)+\dot{V}(t)
\end{aligned}
$$

and

$$
\begin{aligned}
& \rho^{-1}(i) \cdot\left(\tau_{\text {int }}(\sigma(t), V(t)) \cdot H(i)\right. \\
& \left.-2 \cdot \nu \cdot \mathrm{d} H(i) V(t)+2 \cdot \nu \cdot \operatorname{div}_{i} W(i) V(t)\right) \\
& =\mathbf{f}(i)(V(t), V(t)) .
\end{aligned}
$$

In case of $i(M)$ being a large two-sphere in $\mathbb{R}^{3}$ and $\nu$ being small $\nu \cdot \operatorname{Ric}(i)$ may be neglected in this equation. If moreover $\nu=0$ the equation turns over in Euler's equation.

\section{Appendix 1 Geometric Preliminaries}

Let $M$ be as in Sect. 1 and $N$ be a connected, smooth and oriented manifold with a Riemannian metric $($,$) . In case N=\mathbb{R}^{n}$ we write $<,>$ instead of $($,$) and$ mean a fixed scalar product. The Levi-Civita connections of $($,$) and \langle$,$\rangle are$ denoted by $\nabla$ and d respectively. For $J \in E(M, N)$ and $j \in E(\partial M, N)$ we define a Riemannian metric on $M$ and $\partial M$, respectively, by

$$
m(J)(X, Y):=(T J X, T J Y), \quad \forall X, Y \in \Gamma T M
$$

and

$$
m(j)(X, Y):=(T j X, T j Y), \quad \forall X, Y \in \Gamma T \partial M .
$$

One also uses the notations $m(J)=J^{\star}($,$) and m(j)=j^{\star}($,$) . Here \Gamma T Q$ denotes the collection of all smooth vector fields of any smooth manifold $Q$ (with or without boundary). Both $m(J)$ and $m(j)$ depend smoothly on $J$ and $j$.

Associated with the metrics $m(J)$ and $m(j)$, we have the respective Levi-Civita connections $\nabla(J)$ on $M$ and $\nabla(j)$ on $\partial M$. They are determined by

$$
T J \nabla(J)_{X} Y=\nabla_{X}(T J Y)-\left(\nabla_{X}(T J X)\right)^{\perp}, \quad \forall X, Y \in \Gamma T M
$$

and

$$
T j \nabla(j)_{X} Y=T J \nabla(J)_{X} Y-m(j)(W(j) X, Y) \cdot N(j), \forall X, Y \in \Gamma T \partial M .
$$

By $W(j)$ we mean the Weingarten map given as follows: Let $N(j)=T J \cdot \mathbf{n}$, where $\mathbf{n}$ is the positively oriented unit normal vector field (depending on $m(J)$ ), then

$$
\left(\nabla_{Z} N(j)\right)^{\top}=T J \cdot W(j) Z, \quad \forall Z \in \Gamma T M,
$$

where "T" denotes the component tangential to $T J T M$. In case $N=\mathbb{R}^{n}$, we may replace $T J$ and $T j$ by $\mathrm{d} J$ and $\mathrm{d} j$ respectively. For any $J \in E\left(M, \mathbb{R}^{n}\right)$ and any $j \in E\left(\partial M, \mathbb{R}^{n}\right)$ let us denote by $\mu(J)$ and $\mu(j)$ the Riemannian volume form determined by $m(J)$ and the orientation of $M$ respectively by $m(j)$ and the orientation of $\partial M$. We define in case of $N=\mathbb{R}^{n}$ and $\operatorname{dim} \partial M=n-1$ the second 
fundamental form $\mathbf{f}(j)$ by

$$
\mathbf{f}(j)(X, Y)=m(j)(W(j) X, Y)
$$

for all $j \in E\left(\partial M, \mathbb{R}^{n}\right)$ and for all $X, Y \in \Gamma T M$. Moreover $H(j):=\operatorname{Tr} W(j)$ is called here the mean curvature and $\kappa(j):=\operatorname{det} W(j)$ the Gaussian curvature of $j(\partial M) \subset \mathbb{R}^{n}$ respectively. Reference for this section are [1],[2] and [10].

\section{Appendix 2 Computing Force Densities}

$M$ and $N$ are as in Append. 1. Computation of force densities is based on the following proposition:

Proposition A1 Let $\operatorname{dim} M=m \leq \operatorname{dim} N$ and let $K \in C^{\infty}(M, T N)$, then for each $J \in E(M, N)$ and each $L \in C^{\infty}(M, T N)$ the following equation holds :

$$
\int_{M} \nabla K \cdot \nabla L \mu(J)=\int_{M}\left(\Delta(J) K^{-}, L\right) \mu(J)+\int_{\partial M}\left(\nabla_{\mathbf{n}} K, L\right) i_{\mathbf{n}} \mu(J),
$$

where

$\nabla K \cdot \nabla L=-\frac{1}{2} \operatorname{Tr} c(\nabla K(J), T J) \cdot c(\nabla(J) L, T J)+\operatorname{Tr} A(\nabla K(J), T J) \cdot \tilde{A}(\nabla L, T J)$

(with $\nabla$ the Levi-Civita connection of the metric (, ) on $N$ ) and $\Delta(J)$ denotes the Laplacian of $m(J)$.. This implies in turn a Green's equation

$$
\begin{aligned}
& \int_{M}(\Delta(J) K, L) \mu(J)-\int_{M}(K, \Delta(J) L) \mu(J) \\
= & \int_{\partial M}\left(\nabla_{\mathbf{n}} L, K\right) i_{\mathbf{n}} \mu(J)-\int_{\partial M}\left(\nabla_{\mathbf{n}} K, L\right) i_{\mathbf{n}} \mu(J) .
\end{aligned}
$$

Here $i_{\mathrm{n}} \mu(J)$ is the volume element on $\partial M$ defined by $\mu(J)$.

Proof. Writing any $L \in C^{\infty}(M, T N)$ relative to a given $J \in E(M, N)$ in the form

$$
L=T J X(L, J)+L^{\perp},
$$

with a unique $X(L, J) \in \Gamma T M$ (and $L^{\perp}$ being such that $L^{\perp}(p)$ is the component normal to $T J T_{p} M$ for all $p \in M$ ), we have the following formula at hand:

$$
\nabla_{X} L=T J \nabla(J)_{X} X(L, J)+\left(\nabla_{X} L\right)^{\perp}, \quad \forall X \in \Gamma T M .
$$

and write in turn

$$
T_{X} L=c(\nabla L, T J) \cdot T J+T J A(\nabla L, T J) .
$$

From (A2) and (A3) we read off :

$$
c(\nabla L, T) T J=(\nabla L)^{\perp},
$$


as well as

$$
A(\nabla L, T J)=\nabla X(L, J)+W(J, L) \quad \forall L \in C^{\infty}(M, T N) \quad \forall J \in E(M, N) .
$$

Here $W(J, L)$ is given by $T J W(J, L) X=\left(\nabla L^{\perp} X\right)^{\top}$, with " T" denoting the pointwise formed component tangential to $T J T M$. Following [18] we introduce the notions of $\Delta(J) K$ and $\Delta(J) \gamma$, the Laplacian, for any $K \in C_{J}^{\infty}(M, T N)$ and any $\gamma \in A_{J}^{1}(M, T N)$ (where the index $J$ means that $K$ and $\gamma$ respectively cover $J)$. Introducing $\nabla^{\star}$ we set for any $K^{\star} \in C_{J}^{\infty}(M, T N)$, any $\gamma \in A_{J}^{1}(M, T N)$ and any $J \in E(M, N)$

$$
\nabla^{\star} I i:=0
$$

and

$$
\nabla^{\star} \gamma:=-\sum_{r=1}^{m} \nabla(J)_{E_{r}}(\gamma)\left(E_{r}\right),
$$

with $\nabla(J)_{X}(\gamma)(Y)=\nabla_{X}(\gamma Y)-\gamma\left(\nabla(J)_{X} Y\right)$ for any pair $X, Y \in \Gamma T M$. Clearly if $\gamma \in A^{1}(M, \mathbb{R})$ and $\nabla=\mathrm{d}$ then

$$
\mathrm{d}^{\star} \gamma=-\operatorname{div}_{J} Y
$$

provided that $\gamma(X)=m(J)(Y, X)$, for all $X \in \Gamma T M$. The Laplacian $\Delta(J)$ is then defined by

$$
\Delta(J):=\nabla \nabla^{\star}+\nabla^{\star} \nabla
$$

Consequently we have

$$
\Delta(J) K=\nabla^{\star} \nabla K=-\sum_{r=1}^{m} \nabla_{E_{r}}(\nabla K)\left(E_{r}\right) .
$$

For each $L \in T_{J} E(M, N)$ and for each $J \in E(M, N)$ we write

$$
\nabla L=\bar{A}(\nabla L, T J) T J
$$

with $\bar{A}(\nabla L, T J): T N|J(M) \longrightarrow T N| J(M)$ a smooth bundle endomorphism. Then for any moving frame $E_{1}, \ldots, E_{m}$ on $M$ orthonormal with respect to $m(J)$ we deduce

$$
\begin{aligned}
\nabla K \cdot \nabla L & =\sum_{r=1}^{m}\left(\bar{A}^{\star}(\nabla K, T J) \cdot \bar{A}(\nabla L, T J)\left(T J E_{r}\right), T J E_{r}\right) \\
& =\sum_{r=1}^{m}\left(\bar{A}^{\star}(K, T J) \cdot \nabla_{E_{r}} L, T J E_{r}\right),
\end{aligned}
$$

with $\bar{A}^{\star}(\nabla K, T J)$ being the adjoint of $\vec{A}(\nabla K, T J)$ formed with respect to $($,$) .$ Hence

$$
\begin{aligned}
\nabla K \cdot \nabla L & =\sum_{r=1}^{m}\left(\nabla_{E_{r}}\left(\bar{A}^{\star}(\nabla K, T J) L\right), T J E_{r}\right) \\
& -\sum_{r=1}^{m}\left(L, \nabla_{E_{r}}(\bar{A}(\nabla K, T J)) T J E_{r}\right)
\end{aligned}
$$


holds true. We derive from (A4)

$$
\begin{aligned}
\nabla K \cdot \nabla L & =\sum_{i=1}^{m}\left(\nabla_{E_{r}}\left(\bar{A}^{\star}(\nabla K, T J) L\right), T J E_{r}\right) \\
& +(\Delta(J) K, L)+\sum_{i=1}^{m}\left(\bar{A}(\nabla K, T J) \nabla_{E_{r}}(T J) E_{r}, L\right) .
\end{aligned}
$$

Since $\left(\bar{A}^{\star}(\nabla(K, T J) L)^{\top}=T J Z(K, L, J)\right.$ for some well-defined $Z(K, L, J)$ and $\nabla_{E_{r}}(T J) E_{r}$ is pointwise normal to $T J T M$ the following series of equations hold true :

$$
\begin{aligned}
\nabla K \cdot \nabla L & =-\sum_{i=1}^{m}\left(\nabla_{E_{r}}(c(\nabla K, T J) L), T J E_{r}\right)+\operatorname{div}_{J} Z(K, L, J) \\
& +(\Delta(J) K, L)+\sum_{i=1}^{m}\left(c(\nabla K, T J) \nabla_{E_{r}}(T J) E_{r}, L^{\top}\right) \\
& =-\sum_{i=1}^{m}\left(\nabla_{E_{r}}\left(c(\nabla K, T J) L^{\perp}\right), T J E_{r}\right) \\
& -\sum_{i=1}^{m}\left(\nabla_{E_{r}}\left(c(\nabla K, T J) L^{\top}\right), T J E_{r}\right) \\
& +\operatorname{div}_{J} Z(K, L, J)+(\Delta(J) K, L) \\
& +\sum_{i=1}^{m}\left(c(\nabla K, T J) \nabla_{E_{r}}(T J) E_{r}, L^{\top}\right)
\end{aligned}
$$

with $\operatorname{div}_{J}$ the divergence operator associated with $m(J)$.

Writing $c(\nabla K, T J) L^{\perp}=T J U(K, L, J)$, for some well defined $U(K, L, J) \in$ $\Gamma T M$, we obtain

$$
\nabla K \cdot \nabla L=-\operatorname{div}_{J} U(K, L, J)+\operatorname{div}_{J} Z(K, L, J)+(\Delta(J) K, L) .
$$

Here $U(K, L, J)$ is given by $T J U(K, L, J)=c(\nabla K, T J) L^{\perp}$. The theorem of Gauss yields the desired equation (A1).

\section{Appendix 3 Simple Examples}

Here we will study well known one-forms on $E\left(M, \mathbb{R}^{n}\right)$ in the light of our formalism developed above. For simplicity let $\operatorname{dim} M=n$. However, (A5) and (A6) below hold also for $\operatorname{dim} M \leq n$. Again $j:=J \mid \partial M$ and $l:=L \mid \partial M$. The index " 0 " means here the component in $E_{0}\left(M, \mathbb{R}^{n}\right)$.

(i.) In our first example we specify a constitutive map $\mathcal{H}$ by $\mathcal{H}(\mathrm{d} J)=J_{0}$ for all $J \in E\left(M, \mathbb{R}^{n}\right)$, with $J_{0}$ the component of $J$ in $E_{0}\left(M, \mathbb{R}^{n}\right)$. Then if $X(L, J)$ is as in (A2) 


$$
\begin{aligned}
\int_{M} \mathrm{~d} J \cdot \mathrm{d} L \mu(J) & =\int_{M} \operatorname{Tr} \bar{A}(\mathrm{~d} L, \mathrm{~d} J) \mu(J)=\int_{M} \operatorname{Tr} \nabla(J) X(L, J) \mu(J) \\
& =\int_{M} \operatorname{div}_{J} X(L, J) \mu(J)=\int_{\partial M}<N(j), L>i_{\mathrm{n}} \mu(j) \\
& =\mathrm{D}\left(\int_{M} \mu(J)\right)(L)
\end{aligned}
$$

holds for all $J \in E\left(M, \mathbb{R}^{n}\right)$ and for all $L \in C^{\infty}\left(M, \mathbb{R}^{n}\right)$. Moreover $N(j)=$ $T J \mathbf{n}$. Introducing the volume function $\mathcal{V}: E\left(M, \mathbb{R}^{n}\right) \longrightarrow \mathbb{R}$, which assigns to any $J \in E\left(M, \mathbb{R}^{n}\right)$ the volume

$$
\mathcal{V}(J)=\int_{M} \mu(J)
$$

yields

$$
\mathrm{D} \mathcal{V}(J)(L)=\int_{M} \mathrm{~d} J \cdot \mathrm{d} L \mu(J)
$$

Let $h_{\mathbf{n}}$ be given by $\Delta(j) h_{\mathbf{n}}(\mathrm{d} j)=N(j)$ for all $j \in E\left(\partial M, \mathbb{R}^{n}\right)$. Hence

$$
\mathrm{D} \mathcal{V}(J)(L)=\int_{\partial M} \eta_{\mathbf{n}}(\mathrm{d} j) \cdot \mathrm{d} l \mu(j)
$$

$\forall J \in E\left(M, \mathbb{R}^{n}\right)$ and $\forall L \in C^{\infty}\left(M, \mathbb{R}^{n}\right)$.

(ii.) Next let us turn our attention to $\eta_{\partial}$ defined by the formula $\eta_{\partial}(\mathrm{d} j)=j_{0}$ for all $j \in E\left(\partial M, \mathbb{R}^{n}\right)$ with $j_{0} \in E_{0}\left(\partial M, \mathbb{R}^{n}\right)$. For any $J \in E\left(\partial M, \mathbb{R}^{n}\right)$ and any $l \in C^{\infty}\left(\partial M, \mathbb{R}^{n}\right)$ one easily verifies the following (cf. (25)) :

$$
\begin{aligned}
\int_{\partial M}<\Delta(j) j, l>\mu(j) & =\int_{\partial M} \mathrm{~d} j \cdot \mathrm{d} l \mu(j) \\
& =\int_{\partial M}\left(\operatorname{div}_{j} X(l, j)+\theta(l, j) \cdot H(j)\right) \mu(j) \\
& =\int_{\partial M}<H(j) \cdot N(j), l>\mu(j) \\
& =\mathrm{D}\left(\int_{\partial M} \mu(j)\right)(l) .
\end{aligned}
$$

(Thus $\Delta(j) j=H(j) \cdot N(j)$ for each $j \in E\left(\partial M, \mathbb{R}^{n}\right)$.) If we define the area function $\mathcal{A}: E\left(\partial M, \mathbb{R}^{n}\right) \longrightarrow \mathbb{R}$, which sends any $j \in E\left(\partial M, \mathbb{R}^{n}\right)$ into $\mathcal{A}(j)=$ $\int_{\partial M} \mu(j)$ we get

$$
\mathrm{DA}(j)(l)=\int_{\partial M} \mathrm{~d} j \cdot \mathrm{d} l \mu(j)
$$

for all variables of $\mathrm{D} \mathcal{A}$.

(iii.) Next let us consider the map $\hbar_{\partial}^{\prime}: E\left(\partial M, \mathbb{R}^{n}\right) \longrightarrow C^{\infty}\left(\partial M, \mathbb{R}^{n}\right)$ given by $h_{\partial}^{\prime}(\mathrm{d} j)=N(j)$ for all $j \in E\left(\partial M, \mathbb{R}^{n}\right)$. Then the formula 


$$
\Delta(j) N(j)=-\mathrm{d} j \operatorname{grad}_{j} H(j)+\left(\operatorname{Tr} W(j)^{2}\right) \cdot N(j)
$$

holds for any $j \in E\left(\partial M, \mathbb{R}^{n}\right)$. In the special case of $\operatorname{dim} \partial M=2$ a topological constant, the Euler characteristic $\chi(\partial M)$, enters the constitutive law $F$. It is hidden in the virtual work obtained by destorting $\partial M$ in the unit normal direction:

$$
F(j)(N(j))=\int_{\partial M}<\Delta(j) N(j), N(j)>\mu(j)=\int_{\partial M} \operatorname{Tr} W(j)^{2} \mu(j) .
$$

Applying the theorems of Cayley-Hamilton (cf. [11]) and of Gauss-Bonnet (cf. [10]) to the very right hand side of equation (A6) yields :

$$
F(j)(N(j))=-4 \pi \chi(\partial M)+\int_{\partial M} H(j)^{2} \mu(j)
$$

\section{Appendix 4 A Metric on $E(M, N)$ Defined via Mass Density and its Geodesics}

Here $N$ is as in Append. 1. We first introduce a metric $\mathcal{B}$ on $E(M, N)$, of which the geodesics represent free motions. This metric is based on a density map $\rho$ of which we may think as a mass density. A map $\rho \in C^{\infty}\left(E(M, N), C^{\infty}(M, \mathbb{R})\right)$ is called a density map if $\rho(J)(p)>0$ for all $p \in M$ and for $J \in E(M, N)$ and in addition satisfies the continuity equation

$$
\mathbf{d} \rho(J)(L)=-\frac{\rho(J)}{2} \operatorname{Tr}_{m(J)} \mathbf{d} m(J)(L) \quad \forall J \in E(M, N) \text { and } \forall L \in C^{\infty}(M, T N) .
$$

For some simple proof of the existence of density maps we refer to [7]. Here d denotes the differential of maps from Fréchet manifolds into Fréchet spaces and $\dot{\mathrm{d}} m(J)$ is given for all $J \in E(M, N)$ by

$$
\mathrm{d} m(J)(L)(X, Y)=\left(\nabla_{X} L, T J Y\right)+\left(T J X, \nabla_{Y} L\right),
$$

for all $L \in C^{\infty}(M, T N)$ and all $X, Y \in \Gamma T M$, cf. [8]. We now define the metric $\mathcal{B}$ on $E(M, N)$ by

$$
\mathcal{B}(J)\left(L_{1}, L_{2}\right):=\int_{M} \rho(J)\left(L_{1}, L_{2}\right) \mu(J)
$$

for any $J \in E(M, N)$ and any pair $L_{1}, L_{2} \in C_{J}^{\infty}(M, T N)=T_{J} E(M, N)$. Let us next determine the geodesic spray of $\mathcal{B}$. The cotangent bundle of $E(M, N)$ is denoted by $T^{\star} E(M, N)$. For any $J \in E(M, N)$ its fiber $T_{J}^{\star} E(M, N)$ consists of the smooth linear maps from $T_{J} E(M, N)$ into $\mathbb{R}$. The metric $\mathcal{B}$ yields the injective map $\mathcal{B}^{\sharp}$ defined by

$$
\mathcal{B}^{\sharp}(J)(L):=\mathcal{B}(J)(L, . .)=\int_{M} \rho(J)(L, . .) \mu(J)
$$


$\forall L \in C_{J}^{\infty}(M, N)$ and $\forall J \in E(M, N)$. We will work on $\operatorname{Im} \mathcal{B}^{\sharp}$ only (the topology is such that $\mathcal{B}^{\sharp}$ is a diffeomorphism). Let $\Pi^{\star}: \operatorname{Im} \mathcal{B}^{\sharp} \longrightarrow E(M, N)$ be the projection. Then the canonical one-form on $\operatorname{Im} \mathcal{B}^{\sharp}$ is given by

$$
\theta_{E}\left(\mathcal{W}_{\mathcal{B}^{\sharp}(L)}\right)=-\mathcal{B}^{\sharp}(L)\left(T \Pi^{\star} \mathcal{W}_{\mathcal{B}^{\sharp}(L)}\right)
$$

where $\mathcal{W}_{\mathcal{B}^{\sharp}(L)} \in T_{\mathcal{B}^{\sharp}(L)} \operatorname{Im} \mathcal{B}^{\sharp}$ for all $L \in T E(M, N)$ and all $K \in T_{L} T C^{\infty}(M, N)$. Let $C_{L}^{\infty}\left(M, T^{2} N\right):=\left\{K^{-} \in C^{\infty}(M, T N) \mid \pi_{T N} \circ K=L\right\}$, for a given map $L \in$ $\left.C^{\infty}(M, T N)\right)$. Thus for all $K \in C_{L}^{\infty}\left(M, T^{2} N\right)$ for any $L \in C_{J}^{\infty}(M, T N)$ equation (A8) turns into

$$
\left(\mathcal{B}^{\sharp} \theta_{E}\right)(L)(K)=-\int_{M} \rho(J)\left(T \pi_{N} \circ K, L\right) \mu(j) .
$$

Due to the continuity equation (A7) the symplectic form $\omega_{E}$ is given by

$$
\omega_{E}(L)\left(K_{1}, K_{2}\right)=\int_{M} \rho(J) \omega(L)\left(K_{1}, K_{2}\right) \mu(J)
$$

where $\omega:=\mathrm{d} \theta_{N}$ and where $\dot{\theta}_{N}$ is the pull-back (by the metric on $\dot{N}$ ) of the canonical one-form on $T^{\star} N$. Thus the geodesic spray

$$
s: T E(M, N) \longrightarrow T^{2} E(M, N)
$$

is determined by

$$
\omega_{E}(L)(s(L), K)=\mathrm{d} \mathcal{E}(L)(K)
$$

where

$$
\mathcal{E}(L):=\frac{1}{2} \mathcal{B}(L, L), \quad \forall L \in C^{\infty}(M, T N) \text { and } \forall J \in E(M, N) .
$$

The energy e $: T N \longrightarrow \mathbb{R}$ is defined by

$$
e\left(v_{q}\right):=\frac{1}{2}\left(v_{q}, v_{q}\right), \quad \forall v_{q} \in T_{q} N \text { and } \forall q \in N .
$$

Let $e_{\star}$ be given by

$$
\left(\epsilon_{\star}\right)(J)(L)(p):=e(L(p))
$$

for all $L \in C_{J}^{\infty}(M, T N)$, all $J \in E(M, N)$ and all $p \in M$. Since

$$
\mathcal{E}(L)=\int_{M} \rho(J) e_{\star}(L) \mu(J), \quad \forall L \in C_{J}^{\infty}(M, T N) \text { and } \forall J \in E(M, N),
$$

we immediately deduce from (A9) and (A10) the following:

Lemma A2 The geodesic spray $s$ of $\mathcal{B}$ is $s_{\star}: C^{\infty}(M, T N) \longrightarrow C^{\infty}\left(M, T^{2} N\right)$, given by

$$
s_{\star}(L)=s \circ L, \quad \forall L \in C^{\infty}(M, N), \text { and } \forall J \in E(M, N)
$$


E. Binz

where $s: T N \longrightarrow T^{2} N$ is the geodesic spray of the metric (,) on $N$. Hence a geodesic curve $\sigma:(-\lambda, \lambda) \longrightarrow E(M, N)$ with $\lambda \in \mathbb{R}^{+}$and the initial conditions $\sigma(0)=J$ and $\dot{\sigma}(0)=L$ on $E(M, N)$ is uniquely determined by the requirement that $\sigma_{p}:(-\lambda, \lambda) \longrightarrow N$ given by

$$
\sigma_{p}(t):=\sigma(t)(p), \quad \forall t \in(-\lambda, \lambda)
$$

is a geodesic curve in $N$ with the initial data $\sigma_{p}(0)=p$ and $\dot{\sigma}(0)=L(p)$ for any fixed $p \in M$. Clearly if $N=\mathbb{R}^{n}$ and $()=,<,>$, then $\sigma$ is a straight line segment.

One now introduces the covariant derivative of $\mathcal{B}$ in the obvious fashion.

\section{Acknowledgment}

I am indebted to T. Ackermann and G. Schwarz for many helpful discussions.

\section{References}

1. R. Abraham, J.E. Marsden, T. Ratiu: Manifolds, Tensor Analysis, and Applications (Addison Wesley, Global Analysis, 1983)

2. M. Berger, B. Gostiaux: Differential Geometry: Manifolds Curves and Surfaces (Graduate Texts in Mathematics 115, Springer-Verlag, New York, Berlin, Heidelberg, 1987)

3. E. Binz: "On the Notion of the Stress Tensor Associated with $\mathbb{R}^{n}$-invariant Constitutive Laws Admitting Integral Representations", Rep. Math. Phys. 87 49-57 (1989)

4. E. Binz: "Constitutive Laws of Bounded Smoothly Deformable Media", to appear in Proceedings of the Winter School of the Deutsche Physikalische Gesellschaft, ed. by A.Hirshfeld and J.Debrus (Lecture Notes in Physics, Springer-Verlag, Berlin Heidelberg New York, 1991)

5. E. Binz: "Symmetry, Constitutive Laws of Bounded Smoothly Deformable Media, and Neumann Problems", to appear in Symmetries in Science V, ed. by B.Gruber (Plenuum Press)

6. E. Binz, H. Fischer: "On the Manifold of Embeddings of a Closed Manifold", in Proceedings of the Conference on Differential Geometric Methods in Mathematical Physics, Techn. Universität Clausthal, (1978) (Lecture Notes in Physics 134 310324 (1981))

7. E. Binz, G. Schwarz, D. Socolescu: "On a Global Differential Geometric Description of the Motions of Deformable Media", in Infinite Dimensional Manifolds, Groups, and Algebras, Vol. II, ed. by H.D.Doebner, J.Hennig (1990)

8. E. Binz, J. Sniatycki, H.Fischer: Geometry of Classical Fields, Mathematics Studies 154 (North-Holland Verlag, Amsterdam, 1988)

9. A. Frölicher, A. Kriegl: Linear Spaces and Differentiation Theory (John Wiley, Chichester, England, 1988) 
10. W. Greub, S. Halperin, J. Vanstone: Connections, Curvature and Cohomology, Vols. I and II (Acad. Press, New York, 1972-73)

11. W. Greub: Lineare Algebra I, Graduate Texts in Mathematics, Vol. 23 (SpringerVerlag, Berlin, Heidelberg, New York, 1981)

12. M. Epstein, R. Segev: "Differentiable Manifolds and the Principle of Virtual Work in Continuum Mechanics", J. Math. Phys. 21 1243-1245 (1980)

13. E. Hellinger: "Die allgemeinen Ansätze der Mechanik der Kontinua", Enzykl. Math. Wiss. 4/4 (1914)

14. M.W. Hirsch: Differential Topology (Springer GTM, Berlin, 1976)

15. L. Hörmander: Linear Partial Differential Operations, Grundlehren der mathematischen Wissenschaften, Vol.116 (Springer Verlag, Berlin, Heidelberg, New York, 1976)

16. F. John: Partial Differential Equations, (Applied Mathematical Science, Vol.1, 1978)

17. L.D. Landau, E.M. Lifschitz: Elastizitätstheorie, Lehrbuch der theoretischen Physik, Vol. VII (4. Auflage, A kademie Verlag, Berlin, 1975)

18. Y. Matsushima: "Vector Bundle Valued Canonic Forms", Osaka J. Math. 8 309-328 (1971)

19. R. Montgomery: "Isoholonomic Problems and Some Applications", Commun. Math. Phys. 128 562-592 (1990)

20. J. Serrin: Mathematical Principles of Classical Fluid Mechanics, Encyclopedia of Physics, Vol. VIII/1, ed. by C. Truesdell and S.Flügge (Springer-Verlag, Berlin, 1959)

21. M. Spivak: A Comprehensive Introduction to Differential Geometry, Vol. V (Publish and Perish Inc., Boston Ma.)

22: C. Truesdell, W. Noll: The Non-Linear Field Theories of Mechanics, Encyclopedia of Physics, Vol. III/3, ed. by C.Truesdell and S.Flügge (Springer-Verlag, Berlin, 1973)

This book was processed by the author using the $\mathrm{T}_{\mathrm{E}} \mathrm{X}$ macro package from SpringerVerlag. 